\title{
Minimal number of periodic points for smooth self-maps of $S^{3}$
}

\author{
by \\ Grzegorz Graff (Gdańsk) and Jerzy Jezierski (Warszawa)
}

\begin{abstract}
Let $f$ be a continuous self-map of a smooth compact connected and simply-connected manifold of dimension $m \geq 3$ and $r$ a fixed natural number. A topological invariant $D_{r}^{m}[f]$, introduced by the authors [Forum Math. 21 (2009)], is equal to the minimal number of $r$-periodic points for all smooth maps homotopic to $f$. In this paper we calculate $D_{r}^{3}[f]$ for all self-maps of $S^{3}$.
\end{abstract}

1. Introduction. A classical problem in periodic point theory is to determine or estimate the least number of fixed, or more generally $r$-periodic, points in the homotopy class of a given self-map $f$ of a compact manifold $M^{m}$, where $r$ is a fixed natural number (cf. [2], [11]). If $M^{m}$ is simplyconnected and has dimension $m \geq 3$, then one can always find a map $g$ homotopic to $f$ with only one point in $\operatorname{Fix}\left(g^{r}\right)$ (cf. [9]). This is, however, impossible if we demand additionally that $g$ is smooth. In [6] the authors define a topological invariant $D_{r}^{m}[f]$ equal to the minimal number of elements in $\operatorname{Fix}\left(g^{r}\right)$ for all $g$ which are smooth and homotopic to $f$. This leads to a new, smooth branch of Nielsen periodic point theory. Let us remark that $D_{r}^{m}[f]$ may be interpreted purely in terms of the smooth category, namely we may assume that $f$ is smooth and approximate the homotopy which joins $f$ to $g$ by a smooth one. Then $D_{r}^{m}[f]$ gives the minimal number of periodic points in the smooth homotopy class of $f$.

This invariant is obtained by decomposing the Lefschetz numbers of iterations into sequences which can be realized as local fixed point indices of iterations of a $C^{1}$ map at an isolated periodic orbit. As a result, to find $D_{r}^{m}[f]$ we need two types of data: information about $\left\{L\left(f^{n}\right)\right\}_{n \mid r}$ (more precisely, about the set of so-called algebraic periods: $\left\{n \in \mathbb{N}: \sum_{k \mid n} \mu(n / k) L\left(f^{k}\right) \neq 0\right\}$,

2000 Mathematics Subject Classification: Primary 37C25, 55M20; Secondary 37C05.

Key words and phrases: least number of periodic points, indices of iterations, smooth maps, Nielsen number, low dimensional dynamics. 
for $n \mid r$, where $\mu$ is the Möbius function) and the description of all possible sequences of local indices of iterations. The article joins the ideas of three papers: [6] in which $D_{r}^{m}[f]$ is defined, [12] where a description of the algebraic periods of self-maps of $S^{3}$ is given, and finally [7], giving a classification of sequences of local indices of iterations in dimension 3. Basing on these results we are able to determine $D_{r}^{3}[f]$ for all self-maps of a smooth 3-manifold which is closed, connected and simply-connected, i.e., a 3-dimensional sphere, provided that the result of G. Perelman on the Poincare conjecture is true.

It is worth pointing out that $D_{r}^{3}[f]$, for self-maps of $S^{3}$, is almost independent of $f$, namely it is insensitive to the homotopy class of $f$, which seems rather unexpected. For example, if $r$ is odd and $f$ is a map with $|\operatorname{deg}(f)|>1$, then $D_{r}^{3}[f] \in\{\zeta(r)-1, \zeta(r)\}$, where $\zeta(r)$ is the number of divisors of $r$ (cf. Theorem 4.2). This follows from the simply-connectedness of $S^{3}$ and the fact that the set of algebraic periods is equal to the set of all natural numbers. As a consequence, for $S^{3}$ the value of $D_{r}^{3}[f]$ may be perceived as an invariant of the whole space rather than of the homotopy class of $f$. The same remains true for self-maps of 3-dimensional manifolds (with boundary) with fast growth of the Lefschetz numbers of iterations [5].

The article is organized as follows: in Sections 2 and 3 we introduce the notation and definitions and we recall the necessary statements of [6], [7] and [12]. In Section 4 we give the main results (Theorems 4.2 and 4.7). The case of $r$ odd is a consequence of the previously known facts (in particular Theorem 2.9 from [6]), while the case of $r$ even needs a careful and detailed analysis.

2. Preliminary results. A sequence of indices of iterations at an isolated fixed (periodic) point plays a crucial role in minimizing the number of periodic points in a homotopy class. Let $f: U \rightarrow \mathbb{R}^{m}$, where $U$ is an open subset of $\mathbb{R}^{m}$, be a map such that $x_{0}$ is an isolated fixed point for each iteration of $f$. Then the sequence $\left\{\operatorname{ind}\left(f^{n}, x_{0}\right)\right\}_{n=1}^{\infty}$ of local indices is well-defined. Below we introduce a useful notation for representing such sequences, which will be used in the next sections.

Definition 2.1. For a given $k \in \mathbb{N}$ we define

$$
\operatorname{reg}_{k}(n)= \begin{cases}k & \text { if } k \mid n, \\ 0 & \text { if } k \nmid n\end{cases}
$$

In other words, reg $_{k}$ is the periodic sequence

$$
(0, \ldots, 0, k, 0, \ldots, 0, k, \ldots),
$$

where the non-zero entries appear for indices divisible by $k$. A sequence of indices of iterations (just as any integer sequence) has the so-called periodic 
expansion [13],

$$
\operatorname{ind}\left(f^{n}, x_{0}\right)=\sum_{k=1}^{\infty} a_{k} \operatorname{reg}_{k}(n),
$$

where $a_{n}=n^{-1} \sum_{k \mid n} \mu(n / k) \operatorname{ind}\left(f^{k}, x_{0}\right)$ and $\mu$ is the classical Möbius function, i.e., $\mu: \mathbb{N} \rightarrow \mathbb{Z}$ is defined by the following three properties: $\mu(1)=1$, $\mu(k)=(-1)^{s}$ if $k$ is a product of $s$ different primes, and $\mu(k)=0$ otherwise.

It has turned out that the indices of iterations must satisfy some conditions, found in [4], called the Dold relations (or Dold congruences).

THEOREM 2.2 (Dold relations). All coefficients of the periodic expansion of a sequence of indices of iterations are integers, i.e., $a_{k} \in \mathbb{Z}$ in (2.1).

For $p \geq 1$ we define $P_{p}(f)=\operatorname{Fix}\left(f^{p}\right) \backslash \bigcup_{0<n<p} \operatorname{Fix}\left(f^{n}\right)$. If $x \in P_{p}(f)$, then the orbit of $x$ will be called a $p$-orbit.

Now we introduce the notion of a differential Dold sequence in $\mathbb{R}^{m}$ for a $p$-orbit, briefly a $D D^{m}(p)$ sequence. This is a sequence which can be realized as a sequence of indices of iterations on an isolated $p$-orbit for some smooth map in $m$-dimensional space.

Definition 2.3. A sequence of integers $\left\{c_{n}\right\}_{n=1}^{\infty}$ is called a $D D^{m}(p)$ sequence if there is a $C^{1}$ map $\phi: U \rightarrow \mathbb{R}^{m}\left(U\right.$ an open subset of $\left.\mathbb{R}^{m}\right)$ and its isolated $p$-orbit $P$ such that $c_{n}=\operatorname{ind}\left(\phi^{n}, P\right)$. If this equality holds for $n \mid r$, where $r$ is fixed, then the finite sequence $\left\{c_{n}\right\}_{n \mid r}$ will be called a $D D^{m}(p \mid r)$ sequence. The number $p$ will be called the multiplicity of $\left\{c_{n}\right\}_{n}$.

There is a close relation, established in [6], between the minimal number of $r$-periodic points for all smooth maps in a given homotopy class and $D D^{m}(p \mid r)$ sequences (Theorem 2.5 below).

DEFINITION 2.4. Let $\left\{\xi_{n}\right\}_{n \mid r}$ be a sequence of integers satisfying the Dold relations, i.e., its coefficients in the periodic expansion are integers. Assume that we are able to decompose $\left\{\xi_{n}\right\}_{n \mid r}$ as

$$
\xi(n)=c_{1}(n)+\cdots+c_{s}(n),
$$

where $c_{i}$ is a $D D^{m}\left(l_{i} \mid r\right)$ sequence for $i=1, \ldots, s$. Each such decomposition determines the sum of the multiplicities, i.e., the number $l=l_{1}+\cdots+l_{s}$. We define $D_{r}^{m}[\xi]$ to be the smallest $l$ which can be obtained in this way.

Let $\left\{L\left(f^{n}\right)\right\}_{n \mid r}$ be the sequence of the Lefschetz numbers of iterations of $f$. We define $D_{r}^{m}[f]=D_{r}^{m}\left[\left\{L\left(f^{n}\right)\right\}_{n \mid r}\right]$.

THEOREM $2.5([6])$. Let $M$ be a smooth, compact, connected and simplyconnected manifold of dimension $m \geq 3$ and $r \in \mathbb{N}$ a fixed number. For $M$ with nonempty boundary, assume additionally that $f$ has no periodic points 
on the boundary. Then

$$
\min \left\{\# \operatorname{Fix}\left(g^{r}\right): g \text { is } C^{1} \text { and is homotopic to } f\right\}=D_{r}^{m}[f] .
$$

By Definition 2.4 determining $D_{r}^{m}[f]$ requires the knowledge of all $D D^{m}(p)$ sequences. There are strong restrictions on such sequences, found by Chow, Mallet-Paret and Yorke [3].

It is not difficult to observe that in order to obtain any $D D^{m}(p)$ sequence $\left\{d_{n}\right\}_{n}$ it is enough to replace each reg by reg $_{p k}$ in the periodic expansion of some $D D^{m}(1)$ sequence $\left\{c_{n}\right\}_{n}$ (we will say that $\left\{d_{n}\right\}_{n}$ comes from $\left\{c_{n}\right\}_{n}$ ). As a consequence, we will know all $D D^{m}(p)$ sequence if we know all $D D^{m}(1)$ sequences. This is provided in dimension 3 by the following theorem:

TheOREM $2.6([7])$. The complete list of $D D^{3}(1)$ sequences is given below:

(A) $c_{A}(n)=a_{1} \operatorname{reg}_{1}(n)+a_{2} \operatorname{reg}_{2}(n)$,

(B) $c_{B}(n)=\operatorname{reg}_{1}(n)+a_{d} \operatorname{reg}_{d}(n)$,

(C) $c_{C}(n)=-\operatorname{reg}_{1}(n)+a_{d} \operatorname{reg}_{d}(n)$,

(D) $c_{D}(n)=a_{d} \operatorname{reg}_{d}(n)$,

(E) $c_{E}(n)=\operatorname{reg}_{1}(n)-\operatorname{reg}_{2}(n)+a_{d} \operatorname{reg}_{d}(n)$,

(F) $c_{F}(n)=\operatorname{reg}_{1}(n)+a_{d} \operatorname{reg}_{d}(n)+a_{2 d} \operatorname{reg}_{2 d}(n)$, where $d$ is odd,

(G) $c_{G}(n)=\operatorname{reg}_{1}(n)-\operatorname{reg}_{2}(n)+a_{d} \operatorname{reg}_{d}(n)+a_{2 d} \operatorname{reg}_{2 d}(n)$, where d is odd. In all cases $d \geq 3$ and $a_{i} \in \mathbb{Z}$.

In fact, in dimension 3 , to find $D_{r}^{3}[f]$ we only need to know some special $D D^{3}(2)$ sequences, in addition to $D D^{3}(1)$ sequences (see Lemma 2.7).

Let us list three $D D^{3}(2)$ sequences which come from $D D^{3}(1)$ sequences of the form (E), (F) and $(\mathrm{G})$ :

$\left(\mathrm{E}^{\prime}\right) c_{E^{\prime}}(n)=\operatorname{reg}_{2}(n)-\operatorname{reg}_{4}(n)+a_{2 d} \operatorname{reg}_{2 d}(n)$, where $d \geq 3$,

$\left(\mathrm{F}^{\prime}\right) c_{F^{\prime}}(n)=\operatorname{reg}_{2}(n)+a_{2 d} \operatorname{reg}_{2 d}(n)+a_{4 d} \operatorname{reg}_{4 d}(n)$, where $d \geq 3$ is odd,

$\left(\mathrm{G}^{\prime}\right) c_{G^{\prime}}(n)=\operatorname{reg}_{2}(n)-\operatorname{reg}_{4}(n)+a_{2 d} \operatorname{reg}_{2 d}(n)+a_{4 d} \operatorname{reg}_{4 d}(n)$, where $d \geq 3$ is odd.

In all cases $a_{2 d}$ and $a_{4 d}$ are arbitrary integers.

Lemma 2.7 ([6]). Let $f: M \rightarrow M$ be a $C^{1}$ map with $\operatorname{dim} M=3$. Then in the definition of $D_{r}^{3}[f]$ it is enough to consider only $D D^{3}(1 \mid r)$ sequences, i.e., sequences which for $n \mid r$ are of the forms $(\mathrm{A})-(\mathrm{G})$, and $D D^{3}(2 \mid r)$ sequences of the forms $\left(\mathrm{E}^{\prime}\right),\left(\mathrm{F}^{\prime}\right)$ and $\left(\mathrm{G}^{\prime}\right)$.

Let $r$ be a fixed natural number. The sequence of Lefschetz numbers $\left\{L\left(f^{n}\right)\right\}_{n=1}^{\infty}$ also satisfies the Dold relations, so we can write its periodic expansion:

$$
L\left(f^{n}\right)=\sum_{k=1}^{\infty} b_{k} \operatorname{reg}_{k}(n) .
$$


Definition 2.8. We define $B(f)$, the set of algebraic periods of $f$, as $B(f)=\left\{k \in \mathbb{N}: b_{k} \neq 0\right\}$, and $B_{r}(f)$, the set of algebraic periods of $f$ up to level $r$, as $B_{r}(f)=\left\{k \in \mathbb{N}: k \mid r\right.$ and $\left.b_{k} \neq 0\right\}$.

Let us now rewrite the formula (2.2) for $n \mid r$ as

$$
L\left(f^{n}\right)=b_{1} \operatorname{reg}_{1}(n)+b_{2} \operatorname{reg}_{2}(n)+b_{4} \operatorname{reg}_{4}(n)+\sum_{k \in G} b_{k} \operatorname{reg}_{k}(n),
$$

where $b_{1}, b_{2}, b_{4}$ are arbitrary integers, and

$$
G=\left\{k \in \mathbb{N}: k \notin\{1,2,4\} \text { and } b_{k} \neq 0\right\}=B_{r}(f) \backslash\{1,2,4\} .
$$

Let

$$
H=\left\{k \in G: k \text { is odd and } b_{k} \neq 0, b_{2 k} \neq 0\right\} .
$$

In the next section we will use the following result proved in [6].

THEOREM 2.9. If $r$ is odd, then

$$
D_{r}^{3}[f]= \begin{cases}\# G & \text { if }|L(f)| \leq \# G \\ \# G+1 & \text { otherwise. }\end{cases}
$$

If $r$ is even and $r>4$, then

$$
D_{r}^{3}[f] \in[\# G-\# H, \# G-\# H+2] .
$$

3. Algebraic periods. In order to calculate $D_{r}^{3}[f]$ we need to know the periodic expansion of the Lefschetz numbers. Thus, we need to know exactly the set of algebraic periods of $f$ (cf. Definition 2.8). We will base on the description of the algebraic periods for self-maps of $S^{3}$ which is given in [12]. We use homology spaces with rational coefficients.

Let us recall that

$$
H_{i}\left(S^{3}\right)= \begin{cases}\mathbb{Q} & \text { for } i=0,3, \\ 0 & \text { otherwise. }\end{cases}
$$

Let $f: S^{3} \rightarrow S^{3}$. The homomorphism $f_{* 3}: H_{3}\left(S^{3} ; \mathbb{Q}\right) \rightarrow H_{3}\left(S^{3} ; \mathbb{Q}\right)$ is multiplication by a number $\beta \in \mathbb{Z}$, called the degree of $f$ (denoted $\operatorname{deg}(f))$. Fix $r \in \mathbb{N}$. We consider the periodic expansion of $\left\{L\left(f^{n}\right)\right\}_{n=1}^{\infty}$ given by (2.2). Recall that by Definition $2.8, b_{n} \neq 0$ is equivalent to $n \in B(f)$.

Lemma 3.1 ([12, Theorem 1.2]). Let $b_{n}$ denote the $n$th coefficient of the periodic expansion of $\left\{L\left(f^{n}\right)\right\}_{n=1}^{\infty}$. Then:

(a) $b_{1}=1-\beta$.

(b) $b_{2}=0$ if and only if $\beta \in\{0,1\}$.

(c) If $n>2$, then $b_{n}=0$ if and only if $\beta \in\{-1,0,1\}$.

Let $\zeta(r)$ denote the number of divisors of $r$. Assume that $\beta \notin\{-1,0,1\}$. Then, by Lemma 3.1, $B(f)=\mathbb{N}$ and the following proposition holds: 
Proposition 3.2. $B_{r}(f)=\{n \in \mathbb{N}: n \mid r\}$, or equivalently $\# B_{r}(f)=\zeta(r)$.

By Proposition 3.2 and the definition of $G$ we obtain:

Proposition 3.3.

(1) For $r$ odd, $G=B_{r}(f) \backslash\{1\}$, thus $\# G=\zeta(r)-1$.

(2) For $r$ even:

- if $4 \mid r$, then $G=B_{r}(f) \backslash\{1,2,4\}$, thus $\# G=\zeta(r)-3$,

- if $4 \nmid r$, then $G=B_{r}(f) \backslash\{1,2\}$, thus $\# G=\zeta(r)-2$.

Lemma 3.4. If $r$ is even, then $\# H=\eta(r)-1$, where $\eta(r)$ denotes the number of odd divisors of $r$.

Proof. Observe that \#H is the number of pairs $k, 2 k$, where $k \mid r, k>1$ is odd, in $G$. As each natural $2 k$ is also an algebraic period, every odd $k>1$ determines such a pair and thus an element in $H$.

4. Minimal number of periodic points for self-maps of $S^{3}$. The exact determination of the minimal number of $r$-periodic points for all smooth maps homotopic to a map $f: S^{3} \rightarrow S^{3}$ of degree $\beta$ will be given in Proposition 4.1 and Theorems 4.2 and 4.7 below.

Proposition 4.1. For each self-map $f$ of $S^{3}$ we have $L\left(f^{n}\right)=1-\beta^{n}$. This implies the following statements.

- If $\beta=1$, then $L\left(f^{n}\right)=0$ for all $n$, hence $D_{r}^{3}[f]=0$.

- If $\beta=-1$, then $L\left(f^{n}\right)=2 \operatorname{reg}_{1}(n)-\operatorname{reg}_{2}(n)$, hence $D_{r}^{3}[f]=1$ for all $r$, because the Lefschetz numbers of iterations form a sequence of the type (A).

- If $\beta=0$, then $L\left(f^{n}\right)=\operatorname{reg}_{1}(n)$, and analogously to the previous case, $D_{r}^{3}[f]=1$.

In order to find $D_{r}^{3}[f]$ for $f$ such that $|\beta|=|\operatorname{deg}(f)|>1$, we will use Theorem 2.9. For $r$ odd, Theorem 2.9 together with Proposition 3.3(1) and the fact that $L(f)=1-\beta$ immediately give the value of $D_{r}^{3}[f]$ :

Theorem 4.2. For $r$ odd and $|\beta|>1$,

$$
D_{r}^{3}[f]= \begin{cases}\zeta(r)-1 & \text { if } \zeta(r) \geq \beta \geq 2-\zeta(r), \\ \zeta(r) & \text { otherwise. }\end{cases}
$$

4.1. The case of $r$ even. Let

$$
L\left(f^{n}\right)=b_{1} \operatorname{reg}_{1}(n)+b_{2} \operatorname{reg}_{2}(n)+b_{4} \operatorname{reg}_{4}(n)+\sum_{k \in G} b_{k} \operatorname{reg}_{k}(n)=\sum_{i} c_{i}(n)
$$

be a minimal decomposition of Lefschetz numbers, where each $c_{i}$ is a $D D^{3}\left(p_{i} \mid r\right)$ sequence and $\sum_{i} p_{i}=D_{r}^{3}[f]$ (cf. Definition 2.4). Let $\mathcal{A}$ be the set consisting of the sequences $c_{i}$. 
Note that, by Lemma 2.7, each $c_{i}$ has one of the forms $(A)-(G),\left(E^{\prime}\right)-\left(G^{\prime}\right)$, which implies that $p_{i} \leq 2$. Recall that by Theorem 2.9,

$$
D_{r}^{3}[f] \in[\# G-\# H, \# G-\# H+2] \text {. }
$$

Definition 4.3. We will say that a sequence $\psi$ of one of the types (A) $-(\mathrm{G}),\left(\mathrm{E}^{\prime}\right)-\left(\mathrm{G}^{\prime}\right)$ reduces a sequence $b_{k} \mathrm{reg}_{k}$ in the periodic expansion of Lefschetz numbers (4.1) if $k \in B_{r}(f)$ and $a_{k}=b_{k}$, i.e. $b_{k}$ reg $_{k}$ appears in the periodic expansion of $\psi$.

For a self-map $f$ of $S^{3}$, the following lemma holds.

Lemma 4.4. If $|\beta|=|\operatorname{deg}(f)|>1,4 \mid r$ and $r>4$ then each set $\mathcal{A}$ of sequences realizing $D_{r}^{3}[f]$ contains a sequence of one of the types $(\mathrm{B})-(\mathrm{E})$ with the term $a_{4} \mathrm{reg}_{4}\left(a_{4} \neq 0\right)$.

Proof. Suppose that in $\mathcal{A}$ there is no sequence of any of the types (B)-(E) with $a_{4} \mathrm{reg}_{4}$, where $a_{4} \neq 0$. Then $b_{4}=\left(\beta^{2}-\beta^{4}\right) / 4 \leq-3($ as $|\beta|>1)$ implies the existence in $\mathcal{A}$ of at least three sequences:

$$
\gamma_{1}, \gamma_{2}, \gamma_{3}
$$

of the types $\left(\mathrm{G}^{\prime}\right)$ or $\left(\mathrm{E}^{\prime}\right)$, since only these give a negative contribution to $b_{4}$. We will show that this leads to a contradiction with the minimality of $\mathcal{A}$.

For odd $d(d>1,4 d \mid r)$ let us consider the following triple which appears in the formula (4.1):

$$
b_{d} \operatorname{reg}_{d}(n)+b_{2 d} \operatorname{reg}_{2 d}(n)+b_{4 d} \operatorname{reg}_{4 d}(n) .
$$

By Lemma 3.1 each coefficient in (4.4) is non-zero. A $\left(\mathrm{G}^{\prime}\right)$ sequence may be used in $\mathcal{A}$ only if it reduces part of such a triple, namely its last two terms. Then we have to use one (B)-(E) sequence to reduce $b_{d} \mathrm{reg}_{d}$, which makes the contribution of the triple (4.4) to $D_{r}^{3}[f]$ equal to $2+1=3$. On the other hand, we may use $(\mathrm{F})$ or $(\mathrm{G})$ to reduce the first two terms and one $(\mathrm{B})-(\mathrm{E})$ sequence to reduce the last. As a result we get the smaller contribution $1+1=2$.

Suppose that among $\gamma_{1}, \gamma_{2}, \gamma_{3}$ in (4.3) there are three $\left(\mathrm{G}^{\prime}\right)$ 's. Their contribution to $D_{r}^{3}[f]$ is 9 . On the other hand, independently of the values of $b_{1}, b_{2}, b_{4}$, there is a smaller realization: we may reduce these three triples by six other sequences in the way indicated above and $b_{1} \operatorname{reg}_{1}(n)+b_{2} \operatorname{reg}_{2}(n)+$ $b_{4} \mathrm{reg}_{4}(n)$ by one (A) sequence and one (B)-(E) sequence. This gives eight sequences in total, contradicting the minimality.

Similarly, we get a contradiction when we assume that at least one of the three sequences $\gamma_{1}, \gamma_{2}, \gamma_{3}$ is of the type $\left(\mathrm{E}^{\prime}\right)$ (we replace the expression $c_{E^{\prime}}=\operatorname{reg}_{2}(n)-\operatorname{reg}_{4}(n)+a_{2 d} \operatorname{reg}_{2 d}(n)$ which counts with multiplicity 2 with $c_{D}=a_{2 d} \mathrm{reg}_{2 d}(n)$ of multiplicity 1 and repeat the same reasoning as in the case of three $(\mathrm{G})$ sequences). 
COROllary 4.5. Under the assumptions of Lemma 4.4,

$$
D_{r}^{3}[f] \in\{\# G-\# H+1, \# G-\# H+2\} .
$$

Proof. By Theorem 2.9 it is enough to exclude the possibility $D_{r}^{3}[f]=$ $\# G-\# H$. Assume that a set $\mathcal{A}$ of sequences realizes $D_{r}^{3}[f]=\# G-\# H$. By Lemma 4.4 there is a sequence of one of the types (B)-(E) in $\mathcal{A}$ with the

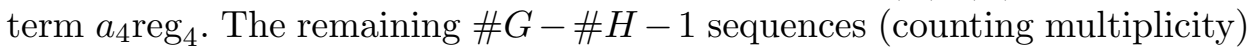
must reduce $b_{k} \mathrm{reg}_{k}$ for $k \in G$. Only (F) and (G) sequences reduce two $b_{k} \mathrm{reg}_{k}$, $k \in G$, and we may use them in such a way $\# H$ times $\left(\left(\mathrm{F}^{\prime}\right),\left(\mathrm{G}^{\prime}\right)\right.$ also reduce two $b_{k} \mathrm{reg}_{k}$ 's but they are counted twice). Now the remaining \#G-2\#H $b_{k} \mathrm{reg}_{k}$ 's must be reduced by $\# G-2 \# H-1$ sequences of types (A)-(E), $\left(\mathrm{E}^{\prime}\right)-\left(\mathrm{G}^{\prime}\right)$ (counting multiplicity). This is impossible, since each of $(\mathrm{A})-(\mathrm{E})$ reduces at most one $b_{k} \mathrm{reg}_{k}$ with $k \in G$, and each $\left(\mathrm{E}^{\prime}\right)-\left(\mathrm{G}^{\prime}\right)$ is counted twice and reduces at most two $b_{k} \operatorname{reg}_{k}$ with $k \in G$.

Lemma 4.6. Suppose that $|\operatorname{deg}(f)|>1$ and $D_{r}^{3}[f]=\# G-\# H+1$. Then there is a minimal set realizing $D_{r}^{3}[f]$ containing only $(\mathrm{B})-(\mathrm{G})$ sequences.

Moreover, we may assume that this set contains one sequence of the type (B)-(E) with $a_{d} \mathrm{reg}_{d}$ such that $d=4$ and $\# G-2 \# H$ sequences of the types (B) $-(\mathrm{E})$ with $d \neq 4$ and \#H sequences of the types $(\mathrm{F})-(\mathrm{G})$.

Proof. Let us fix a minimal set $\mathcal{A}$. By Lemma 4.4 there is a sequence $c_{(4)}$ in $\mathcal{A}$ of one of the types (B)-(E) with $a_{4} \mathrm{reg}_{4}$. We then take $a_{4}=b_{4}$. The remaining sequences realize $b_{k} \operatorname{reg}_{k}$ for $k \in G$, hence each of them must realize at least one $b_{k} \mathrm{reg}_{k}$ so none of them is of the type (A).

Let us assume that $\mathcal{A}$ contains a sequence of the type $\left(\mathrm{G}^{\prime}\right)$,

$$
c_{G^{\prime}}=\operatorname{reg}_{2}-\operatorname{reg}_{4}+a_{2 d} \mathrm{reg}_{2 d}+a_{4 d} \mathrm{reg}_{4 d} .
$$

We will show how to change $\mathcal{A}$ into another minimal system $\mathcal{A}^{\prime}$ in which $c_{G^{\prime}}$ does not appear. Namely, we change three sequences in $\mathcal{A}$ :

- Instead of $c_{G^{\prime}}$ we take two sequences of the type (D): $a_{2 d} \mathrm{reg}_{2 d}, a_{4 d} \mathrm{reg}_{4 d}$.

- Instead of $c_{(4)}$ with $a_{4} \mathrm{reg}_{4}$ we take $c_{(4)}^{\prime}$ with $\left(a_{4}-1\right) \mathrm{reg}_{4}$.

- Let us notice that $b_{2}=\left(\beta-\beta^{2}\right) / 2<0$ implies the existence of a sequence $\psi$ of the type (E) or $(\mathrm{G})$, since only these have a negative contribution to $b_{2}$. Instead of such a sequence we take $\psi^{\prime}(n)=\operatorname{reg}_{2}(n)+\psi(n)$, which is an expression of the type $(\mathrm{B})$ or $(\mathrm{F})$ respectively.

This gives a system $\mathcal{A}^{\prime}$ with the same sum of multiplicities as $\mathcal{A}$ (so also minimal) with one fewer expression of the type $\left(\mathrm{G}^{\prime}\right)$. Similarly we may remove expressions of the type $\left(\mathrm{E}^{\prime}\right)$ and $\left(\mathrm{F}^{\prime}\right)$.

TheOREM 4.7. Let $r$ be even and $|\beta|>1$. Then $D_{r}^{3}[f] \in\{\zeta(r)-\eta(r)-1$, $\zeta(r)-\eta(r)\}$. What is more, $D_{r}^{3}[f]=\zeta(r)-\eta(r)-1$ if and only if one of 
the following two conjunctions holds:

$$
\begin{aligned}
\text { (i) } \quad \zeta(r)-\eta(r) \geq \beta^{2} \quad \text { and } \quad & \left(\beta^{2}-\beta\right) / 2-\eta(r) \geq 0, \\
\text { (ii) } \quad \zeta(r)-3 \eta(r) \geq \beta-2 \quad \text { and } \quad & \left(\beta^{2}-\beta\right) / 2-\eta(r) \leq-1 .
\end{aligned}
$$

Before giving the proof of the above theorem, we illustrate it by the following example:

ExAmple 4.8. Let $f: S^{3} \rightarrow S^{3}$ have degree $\beta=2$ and let $r=12$. The Lefschetz numbers $L\left(f^{n}\right)$ are equal to $1-2^{n}$. We represent this sequence (for $n \mid 12$ ) in the form of a periodic expansion. We get $b_{1}=1-\beta=-1$, $b_{2}=\left(\beta-\beta^{2}\right) / 2=-1, b_{4}=\left(\beta^{2}-\beta^{4}\right) / 4=-3$, thus

$$
\begin{aligned}
L\left(f^{n}\right)= & -\operatorname{reg}_{1}(n)-\operatorname{reg}_{2}(n)-3 \operatorname{rreg}_{4}(n) \\
& +b_{3} \operatorname{reg}_{3}(n)+b_{6} \operatorname{reg}_{6}(n)+b_{12} \operatorname{reg}_{12}(n) .
\end{aligned}
$$

We have $\zeta(12)=6$ and $\eta(12)=2$. Because $\zeta(r)-3 \eta(r)=\beta-2=0$ and $\left(\beta^{2}-\beta\right) / 2-\eta(r)=-1$, we see that the condition (ii) of (4.5) is satisfied and thus $D_{r}^{3}[f]=\zeta(r)-\eta(r)-1=3$. Indeed, we may take the following three sequences, which together realize $\left\{L\left(f^{n}\right)\right\}_{n \mid 12}$ :

$$
\begin{aligned}
c_{G}(n) & =\operatorname{reg}_{1}(n)-\operatorname{reg}_{2}(n)+b_{3} \operatorname{reg}_{3}(n)+b_{6} \operatorname{reg}_{6}(n), \\
c_{C_{1}}(n) & =-\operatorname{reg}_{1}(n)-3 \operatorname{reg}_{4}(n), \\
c_{C_{2}}(n) & =-\operatorname{reg}_{1}(n)+b_{12} \operatorname{reg}_{12}(n) .
\end{aligned}
$$

Proof of Theorem 4.7. First notice that if $|\beta|>1$, then $D_{2}^{3}[f]=1$ and $D_{4}^{3}[f]=2$, thus we verify directly that for $r=2$ and $r=4$ the assertion holds. For $r>4$ we divide the proof into two cases.

PART (I): $4 \mid r$. By Corollary $4.5, D_{r}^{3}[f] \in\{\# G-\# H+1, \# G-\# H+2\}$, which by Proposition 3.3(2) for $4 \mid r$ and Lemma 3.4 is equal to $\{\zeta(r)-\eta(r)-1$, $\zeta(r)-\eta(r)\}$. We will now find conditions equivalent to the statement that $D_{r}^{3}[f]=\# G-\# H+1=\zeta(r)-\eta(r)-1$.

By Lemma 4.6 we may assume that there is a minimal set $\mathcal{A}$ of sequences realizing $D_{r}^{3}[f]$ with \#H sequences of the types $(\mathrm{F})$ or $(\mathrm{G})$ and $\# G-2 \# H$ sequences of the types (B)-(F), which reduce $b_{k} \mathrm{reg}_{k}$ for $k \neq 4$, and one extra expression $c_{(4)}$ of the type (B), (C), (D) or (E), which reduces $b_{4} \mathrm{reg}_{4}$. Thus there are $\# G-\# H$ sequences plus one extra in $\mathcal{A}$.

Let us denote the contribution of the single sequence $c_{(4)}$ to the first two terms of the formula (4.1) by $\epsilon_{1} \operatorname{reg}_{1}(n)+\epsilon_{2} \operatorname{reg}_{2}(n)$, where $\left(\epsilon_{1}, \epsilon_{2}\right) \in$ $\{(1,0),(-1,0),(0,0),(1,-1)\}$.

Let $m_{X}$, where $X \in\{B, C, D, E, F, G\}$, denote the number of sequences (not counting $c_{(4)}$ ) of the given type in the minimal realization $\mathcal{A}$. Then $D_{r}^{3}[f]=\# G-\# H+1$ if and only if there are integers

$$
\left(\epsilon_{1}, \epsilon_{2}\right) \in\{(1,0),(-1,0),(0,0),(1,-1)\}
$$


such that the following system of equations has an integer solution in nonnegative unknowns $m_{X}$ :

$$
\begin{aligned}
m_{B}+m_{C}+m_{D}+m_{E}+m_{F}+m_{G} & =\# G-\# H, \\
m_{B}-m_{C}+m_{E}+m_{F}+m_{G} & =b_{1}-\epsilon_{1}, \\
-m_{E}-m_{G} & =b_{2}-\epsilon_{2}, \\
m_{F}+m_{G} & =\# H,
\end{aligned}
$$

where the first and last equations describe the number of sequences (not counting $c_{(4)}$ ), and the second and third give their contribution to the first two terms of the periodic expansion.

The above system is equivalent to

$$
\begin{aligned}
m_{D}+m_{B}+m_{F}+m_{G} & =\# G-\# H-m_{C}-m_{E}, \\
m_{B}+m_{F}+m_{G} & =b_{1}+m_{C}-m_{E}-\epsilon_{1}, \\
m_{F}+m_{G} & =\# H \\
m_{G} & =-b_{2}-m_{E}+\epsilon_{2} .
\end{aligned}
$$

Notice that:

- For any fixed $m_{C}, m_{E},(4.8)$ is a Cramer system with determinant +1 , thus $m_{D}, m_{B}, m_{F}, m_{G}$ are uniquely determined.

- If $m_{C}, m_{E}$ are integers, then the other unknowns must be integers.

- For any fixed values of $m_{C}, m_{E} \geq 0$ the solutions of (4.8) are nonnegative if and only if the following system of inequalities holds:

$$
\begin{aligned}
0 & \leq-b_{2}-m_{E}+\epsilon_{2} \leq \# H \leq b_{1}+m_{C}-m_{E}-\epsilon_{1} \\
& \leq \# G-\# H-m_{C}-m_{E} .
\end{aligned}
$$

As a consequence, to find the solution of the system (4.7), it is enough to solve (4.9) with integer $m_{C}, m_{E}$ such that $m_{C}, m_{E} \geq 0$. We rewrite (4.9) as a system of four inequalities:

$$
\begin{aligned}
m_{E} & \leq-b_{2}+\epsilon_{2}, \\
m_{E} & \geq-b_{2}+\epsilon_{2}-\# H, \\
m_{C}-m_{E} & \geq \# H-b_{1}+\epsilon_{1}, \\
m_{C} & \leq \frac{1}{2}\left(-b_{1}+\# G-\# H+\epsilon_{1}\right) .
\end{aligned}
$$

The problem reduces to finding a point $\left(m_{C}, m_{E}\right) \in \mathbb{Z}^{2}$ with $m_{C}, m_{E} \geq 0$ for which the inequalities (4.10)-(4.13) are satisfied.

We substitute the values of $\# G$ and \#H using Lemma 3.3(2) and Lemma 3.4 and the values of $b_{1}$ and $b_{2}$ calculated directly:

$$
\# G=\zeta(r)-3, \quad \# H=\eta(r)-1, \quad b_{1}=1-\beta, \quad b_{2}=\frac{1}{2}\left(\beta-\beta^{2}\right) .
$$


Then the inequalities (4.10)-(4.13) can be rewritten (in a different order) as

$$
\begin{aligned}
m_{C} & \leq \frac{1}{2}\left(\zeta(r)-\eta(r)+\beta-3+\epsilon_{1}\right), \\
m_{E} & \geq \frac{1}{2}\left(\beta^{2}-\beta\right)-\eta(r)+1+\epsilon_{2}, \\
m_{E} & \leq \frac{1}{2}\left(\beta^{2}-\beta\right)+\epsilon_{2}, \\
m_{E} & \leq m_{C}-\eta(r)-\beta+2-\epsilon_{1} .
\end{aligned}
$$

Now the problem transforms into the following: for which $r \in \mathbb{N}$ and $\beta \in \mathbb{Z}(4|r,| \beta \mid \geq 2)$ can one choose $\left(\epsilon_{1}, \epsilon_{2}\right) \in\{(0,0),(1,0),(-1,0),(1,-1)\}$ so that the inequalities (4.14)-(4.17) have a nonnegative integer solution $\left(m_{C}, m_{E}\right)$ ? To simplify the notations we write

$$
\begin{aligned}
b & =\frac{1}{2}\left(\zeta(r)-\eta(r)+\beta-3+\epsilon_{1}\right), \\
c & =\frac{1}{2}\left(\beta^{2}-\beta\right)-\eta(r)+1+\epsilon_{2}, \\
d & =\frac{1}{2}\left(\beta^{2}-\beta\right)+\epsilon_{2}, \\
e & =-\eta(r)-\beta+2-\epsilon_{1} .
\end{aligned}
$$

Now the system of inequalities (4.14)-(4.17) takes the form

$$
m_{C} \leq b, \quad c \leq m_{E}, \quad m_{E} \leq d, \quad m_{E} \leq m_{C}+e .
$$

Lemma 4.9. Let $b, c, d, e \in \mathbb{R}$ satisfy $c, e \in \mathbb{Z}$ and $c \leq d$. Then the inequalities (4.18) have a nonnegative integer solution $\left(m_{C}, m_{E}\right)$ if and only if $b \geq 0, d \geq 0$, and $\max \{c, 0\} \leq[b]+e$, where $[b]$ denotes the integer part of $b$.

To prove the above lemma it is enough to notice that the first three inequalities describe $(-\infty, b] \times[c, d]$ while the last defines the closed halfplane under the line $m_{E}=m_{C}+e$ in $\left(m_{C}, m_{E}\right)$ coordinates.

Finally, by Lemma 4.9 the problem becomes: for which $\beta$ and $r$ can one choose $\left(\epsilon_{1}, \epsilon_{2}\right)$ such that the following inequalities hold:

$$
\begin{gathered}
\zeta(r)-\eta(r)+\beta-3+\epsilon_{1} \geq 0, \\
\frac{1}{2}\left(\beta^{2}-\beta\right)+\epsilon_{2} \geq 0, \\
\max \left\{\frac{1}{2}\left(\beta^{2}-\beta\right)-\eta(r)+1+\epsilon_{2}, 0\right\} \\
\leq\left[\frac{1}{2}\left(\zeta(r)-\eta(r)+\beta-3+\epsilon_{1}\right)\right]-\eta(r)-\beta+2-\epsilon_{1} .
\end{gathered}
$$


We notice that the inequality (4.20) always holds. In fact, $|\beta| \geq 2$ implies $\beta^{2}-\beta \geq 2$ and $\frac{1}{2}\left(\beta^{2}-\beta\right)+\epsilon_{2} \geq 1-1=0$.

Now we study (4.19). We will consider four cases.

CASE (1): $\zeta(r)-\eta(r)+\beta \leq 1$. Then the inequality (4.19) never holds, hence the system has no solution.

CASE (2): $\zeta(r)-\eta(r)+\beta=2$ and CASE $(3): \zeta(r)-\eta(r)+\beta=3$ will be discussed separately below.

CAse (4): $\zeta(r)-\eta(r)+\beta \geq 4$. Then (4.19) holds for each $\epsilon_{1}$.

We will consider these cases (in reverse order: starting from Case 4 to Case 1) as assumptions in the next subcases. We will look for solutions of the inequality (4.21).

CASE (4). We assume that $\zeta(r)-\eta(r)+\beta \geq 4$ (the inequality (4.19) holds for each $\epsilon_{1}$ ). To get rid of the maximum and the integer part in (4.21) we consider several subcases.

$\operatorname{SubCASE}(4 . \geq): \frac{1}{2}\left(\beta^{2}-\beta\right)-\eta(r) \geq 0$. Now $c=\frac{1}{2}\left(\beta^{2}-\beta\right)-\eta(r)+1+\epsilon_{2}$ $\geq 0$, since $\epsilon_{2} \in\{-1,0\}$. The inequality (4.21) takes the form $\frac{1}{2}\left(\beta^{2}-\beta\right)-\eta(r)+1+\epsilon_{2} \leq\left[\frac{1}{2}\left(\zeta(r)-\eta(r)+\beta-3+\epsilon_{1}\right)\right]-\eta(r)-\beta+2-\epsilon_{1}$, or

$$
\frac{1}{2}\left(\beta^{2}+\beta\right)-1+\epsilon_{1}+\epsilon_{2} \leq\left[\frac{1}{2}\left(\zeta(r)-\eta(r)+\beta-3+\epsilon_{1}\right)\right] .
$$

If the above inequality holds for some $\left(\epsilon_{1}, \epsilon_{2}\right) \in\{(0,0),(1,0),(-1,0),(1,-1)\}$ then it also holds for $(-1,0)$, thus it is enough to solve

$$
\frac{1}{2}\left(\beta^{2}+\beta\right)-2 \leq\left[\frac{1}{2}(\zeta(r)-\eta(r)+\beta-4)\right],
$$

hence

$$
\frac{1}{2}\left(\beta^{2}+\beta\right) \leq\left[\frac{1}{2}(\zeta(r)-\eta(r)+\beta)\right] .
$$

SubsubCASE (4. $\geq$.even): $\zeta(r)-\eta(r)+\beta$ is even. Now we may omit the integer part:

$$
\frac{1}{2}\left(\beta^{2}+\beta\right) \leq \frac{1}{2}(\zeta(r)-\eta(r)+\beta),
$$

which implies

$$
\beta^{2} \leq \zeta(r)-\eta(r) .
$$

Subsubcase (4. $\geq$.odd): $\zeta(r)-\eta(r)+\beta$ is odd. Now we get

$$
\frac{1}{2}\left(\beta^{2}+\beta\right) \leq \frac{1}{2}(\zeta(r)-\eta(r)+\beta)-\frac{1}{2},
$$

which implies

$$
\beta^{2} \leq \zeta(r)-\eta(r)-1 .
$$


Moreover, we notice that in this subsubcase the above inequality is equivalent to

$$
\beta^{2} \leq \zeta(r)-\eta(r)
$$

In fact, by the parity assumption in this subsubcase we have $\zeta(r)-\eta(r)+\beta$ $\equiv 1(\bmod 2)$, and thus $\zeta(r)-\eta(r)-\beta^{2} \equiv 1(\bmod 2)$. As a consequence, the equality $\beta^{2}=\zeta(r)-\eta(r)$ cannot hold.

Thus the assumptions of Case 4, Subcase (4. $\geq$ ) and the above inequality give the following system of conditions:

$$
\zeta(r)-\eta(r)+\beta \geq 4, \quad \frac{\beta^{2}-\beta}{2}-\eta(r) \geq 0, \quad \zeta(r)-\eta(r) \geq \beta^{2} .
$$

Subcase $(4 .<): \frac{1}{2}\left(\beta^{2}-\beta\right)-\eta(r)<0$. Now $c=\frac{1}{2}\left(\beta^{2}-\beta\right)-\eta(r)+1+\epsilon_{2}$ $\leq 0$ (for any $\epsilon_{2}$ ), hence the inequality (4.21) becomes

$$
0 \leq\left[\frac{1}{2}\left(\zeta(r)-\eta(r)+\beta-3+\epsilon_{1}\right)\right]-\eta(r)-\beta+2-\epsilon_{1} .
$$

Let us notice that if the above inequality holds for some $\epsilon_{1} \in\{-1,0,1\}$ then it also holds for $\epsilon_{1}=-1$, hence we get

$$
\eta(r)+\beta-3 \leq\left[\frac{1}{2}(\zeta(r)-\eta(r)+\beta)-2\right] .
$$

Subsubcase $(4 .<$.even $): \zeta(r)-\eta(r)+\beta$ is even. Now $\eta(r)+\beta-3 \leq$ $\frac{1}{2}(\zeta(r)-\eta(r)+\beta)-2$ or

$$
\zeta(r)-3 \eta(r) \geq \beta-2 .
$$

Subsubcase $(4 .<$.odd $): \zeta(r)-\eta(r)+\beta$ is odd. Now $\eta(r)+\beta-3 \leq$ $\frac{1}{2}(\zeta(r)-\eta(r)+\beta)-2-\frac{1}{2}$, hence we get

$$
\zeta(r)-3 \eta(r) \geq \beta-1 .
$$

Moreover, in this subsubcase the above inequality is equivalent to

$$
\zeta(r)-3 \eta(r) \geq \beta-2 .
$$

In fact, the equality $\zeta(r)-3 \eta(r)=\beta-1$ cannot hold because of the parity assumptions in $(4 .<$.odd $)$.

Thus, Case 4, Subcase $(4 .<)$ and the above inequality give the following system of conditions:

$$
\zeta(r)-\eta(r)+\beta \geq 4, \quad \frac{\beta^{2}-\beta}{2}-\eta(r) \leq-1, \quad \zeta(r)-3 \eta(r) \geq \beta-2 .
$$

Case (3): $\zeta(r)-\eta(r)+\beta=3$. The assumption of Case 3 implies that

$$
b=\frac{1}{2}\left(\zeta(r)-\eta(r)+\beta-3+\epsilon_{1}\right)=\frac{1}{2} \epsilon_{1} .
$$


Now $b \geq 0$ for $\epsilon_{1}=0$ or $\epsilon_{1}=1$.

Subcase (3.0): $\epsilon_{1}=0$. Since $\epsilon_{1}=0$ implies $\epsilon_{2}=0$, the inequality (4.21) takes the form

$$
\max \left\{\frac{1}{2}\left(\beta^{2}-\beta\right)-\eta(r)+1,0\right\} \leq-\eta(r)-\beta+2 .
$$

SubsubCASE $(3.0 . \geq): \frac{1}{2}\left(\beta^{2}-\beta\right)-\eta(r)+1 \geq 0$. Now we get

$$
\frac{1}{2}\left(\beta^{2}-\beta\right)-\eta(r)+1 \leq-\eta(r)-\beta+2,
$$

which is equivalent to

$$
\beta^{2}+\beta \leq 2
$$

and the last holds only for $\beta=-2$. Then (3.0. $\geq$ ) and the assumptions of Case (3) take the form $4 \geq \eta(r)$ and $\zeta(r)=\eta(r)+5$ respectively. Since $\eta(r) \mid \zeta(r)$, we obtain $\eta(r)=1$ and $\zeta(r)=6$. This implies $r=2^{5}$.

SubsubCASE $(3.0 .<): \frac{1}{2}\left(\beta^{2}-\beta\right)-\eta(r)+1<0$. We get

$$
0 \leq-\eta(r)-\beta+2 \text {. }
$$

In other words, $\eta(r) \leq-\beta+2$. On the other hand, the assumption $(3.0 .<)$ gives $\beta^{2}-\beta<2 \eta(r)-2$. The above inequalities imply $\beta^{2}+\beta-2<0$, which is never true for $|\beta|>1$.

SubCASE (3.1): $\epsilon_{1}=1$. The inequality (4.21) takes the form

$$
\max \left\{\frac{1}{2}\left(\beta^{2}-\beta\right)-\eta(r)+1+\epsilon_{2}, 0\right\} \leq-\eta(r)-\beta+1 .
$$

Since $\epsilon_{2}$ may be 0 or -1 , here we may put $\epsilon_{2}=-1$, which implies

$$
\max \left\{\frac{1}{2}\left(\beta^{2}-\beta\right)-\eta(r), 0\right\} \leq-\eta(r)-\beta+1 .
$$

$\operatorname{SubsubCASE}(3.1 . \geq): \frac{1}{2}\left(\beta^{2}-\beta\right)-\eta(r) \geq 0$. Now

$$
\frac{1}{2}\left(\beta^{2}-\beta\right)-\eta(r) \leq-\eta(r)-\beta+1
$$

implies $\beta^{2}+\beta-2 \leq 0$, hence $\beta=-2$. Then the assumption of Case 3, $\zeta(r)-\eta(r)+\beta=3$, gives $\zeta(r)=\eta(r)+5$. On the other hand, the condition (3.1. $\geq$ ) takes the form $3 \geq \eta(r)$. Again $\eta(r) \mid \zeta(r)$ implies $\eta(r)=1, \zeta(r)=6$ and thus $r=2^{5}$.

Subsubcase (3.1. $<): \frac{1}{2}\left(\beta^{2}-\beta\right)-\eta(r)<0$. The inequality (4.21) takes the form

$$
0 \leq-\eta(r)-\beta+1
$$


hence implies $\eta(r) \leq-\beta+1$. We combine this inequality with the condition $(3.1 .<)$ to get

$$
\frac{1}{2}\left(\beta^{2}-\beta\right)<\eta(r) \leq-\beta+1 .
$$

This implies the inequality

$$
\frac{1}{2}\left(\beta^{2}-\beta\right)<-\beta+1,
$$

which is not valid for any $|\beta|>1$.

CASE (2): $\zeta(r)-\eta(r)+\beta=2$. Here we get

$$
b=\frac{1}{2}\left(\zeta(r)-\eta(r)+\beta-3+\epsilon_{1}\right)=\frac{1}{2}\left(\epsilon_{1}-1\right) .
$$

Notice that $b \geq 0$ only for $\epsilon_{1}=+1$, and then $b=0$. This shows that (4.21) has the form

$$
\max \left\{\frac{1}{2}\left(\beta^{2}-\beta\right)-\eta(r)+1+\epsilon_{2}, 0\right\} \leq-\eta(r)-\beta+1 .
$$

Since $\epsilon_{2}$ may be 0 or -1 , it is enough to consider $\epsilon_{2}=-1$. We then get

$$
\max \left\{\frac{1}{2}\left(\beta^{2}-\beta\right)-\eta(r), 0\right\} \leq-\eta(r)-\beta+1 .
$$

Subcase $(2 . \geq): \frac{1}{2}\left(\beta^{2}-\beta\right)-\eta(r) \geq 0$. The inequality (4.21) takes the form

$$
\frac{1}{2}\left(\beta^{2}-\beta\right)-\eta(r) \leq-\eta(r)-\beta+1,
$$

which implies $\beta^{2}+\beta \leq 2$, hence $\beta=-2$. Moreover, (2. $\geq$ ) and the condition of Case $(2)$ become $3 \geq \eta(r)$ and $\zeta(r)=\eta(r)+4$ respectively. Since $\eta(r) \mid \zeta(r)$, there are two possibilities: $\eta(r)=1$ or $\eta(r)=2$. In the first case $\zeta(r)=5$ and $r=2^{4}$. In the second case $\zeta(r)=6$ and $r=2^{2} p$ where $p$ is a prime number greater than 2 . Now we get either

- $\beta=-2$ and $r=2^{4}$, or

- $\beta=-2$ and $r=4 p$ for an odd prime $p$.

SubCASE $(2 .<): \frac{1}{2}\left(\beta^{2}-\beta\right)-\eta(r)<0$. Now the inequality $(4.21)$ takes the form $0 \leq-\eta(r)-\beta+1$, which implies $\eta(r) \leq-\beta+1$. On the other hand, the condition $(2 .<)$ gives $\beta^{2}-\beta<2 \eta(r)$. This implies $\beta^{2}+\beta<2$, which has no solution for $|\beta|>1$.

Summing up the previous considerations, we conclude that $D_{r}^{3}[f]=$ $\zeta(r)-\eta(r)-1$ if and only if one of the following five conditions holds (cf. 
(4.22), (4.23), Cases (3) and (2)):

$$
\begin{aligned}
& \text { (1) } \quad \zeta(r)-\eta(r)+\beta \geq 4, \quad \zeta(r)-\eta(r) \geq \beta^{2}, \quad \frac{\beta^{2}-\beta}{2}-\eta(r) \geq 0, \\
& (2) \quad \zeta(r)-\eta(r)+\beta \geq 4, \quad \zeta(r)-3 \eta(r) \geq \beta-2, \quad \frac{\beta^{2}-\beta}{2}-\eta(r) \leq-1,
\end{aligned}
$$

(3) $\beta=-2$ and $r=16$,

(4) $\beta=-2$ and $r=32$,

(5) $\beta=-2$ and $r=4 p$ for an odd prime $p$.

We now show that

$$
[(\mathrm{i}) \text { or }(\mathrm{ii})] \Leftrightarrow[(1) \text { or }(2) \text { or }(3) \text { or }(4) \text { or }(5)] \text {, }
$$

where (i) and (ii) are the conditions of Theorem 4.5, which we recall below:

(i) $\zeta(r)-\eta(r) \geq \beta^{2} \quad$ and $\quad\left(\beta^{2}-\beta\right) / 2-\eta(r) \geq 0$,

(ii) $\zeta(r)-3 \eta(r) \geq \beta-2$ and $\left(\beta^{2}-\beta\right) / 2-\eta(r) \leq-1$.

First we prove that (ii) $\Leftrightarrow(2)$.

$\Leftarrow$ is trivial. To prove $\Rightarrow$ it suffice to show that the second and third inequalities in (2) imply the first. The second and the third inequality give respectively $\zeta(r)-\eta(r) \geq 2 \eta(r)+\beta-2$ and $\eta(r) \geq\left(\beta^{2}-\beta\right) / 2+1$. This implies that $\zeta(r)-\eta(r) \geq \beta^{2}$, and as $\beta^{2} \geq 4-\beta$ for $\beta \neq-2$, we see that the first inequality results from the second and the third for $\beta \neq-2$. If $\beta=-2$, we get $\eta(r) \geq 4$ and we should check whether $\zeta(r)-\eta(r) \geq 2 \eta(r)-4$ implies $\zeta(r)-\eta(r) \geq 6$. This is obviously satisfied if $\eta(r)>4$, and for $\eta(r)=4$ we find (because $4 \mid r$ ) that $\zeta(r)$ must be greater than 10 , so $\zeta(r)-\eta(r) \geq 6$ is also satisfied in this case.

It remains to show that

$$
\text { (i) } \Leftrightarrow[(1) \text { or }(3) \text { or }(4) \text { or }(5)] \text {. }
$$

$\Leftarrow(1)$ implies (i) in a trivial way. Then we check case by case that each of (3), (4), (5) implies (i).

$\Rightarrow$ We show that (i) and the negation of (1) imply the alternative [(3) or $(4)$ or $(5)]$.

Recall that (i) means

$$
\zeta(r)-\eta(r) \geq \beta^{2} \quad \text { and } \quad \frac{\beta^{2}-\beta}{2}-\eta(r) \geq 0 .
$$

Now the negation of (1) means in particular that

$$
\zeta(r)-\eta(r)+\beta<4 .
$$

The above two inequalities imply $\beta^{2} \leq \zeta(r)-\eta(r)<4-\beta$, hence $\beta^{2}+\beta-4$ $<0$, which holds only for $\beta=-2$. This in turn implies that the above 
inequalities take the forms

$$
\zeta(r)-\eta(r) \geq 4, \quad \eta(r) \leq 3, \quad \zeta(r)-\eta(r)<6 .
$$

Now we get $(\zeta(r)-\eta(r)=4$ or 5$)$ and $\eta(r) \leq 3$.

If $\zeta(r)-\eta(r)=5$ then $\eta(r)=1$, hence $r=2^{5}=32$, so we get (4).

If $\zeta(r)-\eta(r)=4$ then $\eta(r)=1$ or 2 . For $\eta(r)=1$ we get $r=2^{4}=16$, so we obtain (3). Finally, for $\eta(r)=2$, we obtain $r=4 p$, where $p$ is an odd prime, which gives (5). This ends the proof of Part I.

PART (II): $2 \mid r$ but $4 \nmid r$. Since $b_{4}=0$, we see that $D_{r}^{3}[f] \in\{\# G-\# H$, $\# G-\# H+1\}=\{\zeta(r)-\eta(r)-1, \zeta(r)-\eta(r)\}$, where the last equality results from Lemma 3.4 and Proposition 3.3(2) for $4 \nmid r$.

Searching for conditions equivalent to $D_{r}^{3}[f]=\# G-\# H$, we repeat the same reasoning as in the case $4 \mid r$, with the only difference being that we do not need an additional sequence to reduce $b_{4} \mathrm{reg}_{4}$, so we have to find nonnegative integer solutions of (4.7) without parameters $\epsilon_{1}, \epsilon_{2}$.

Then similar computations give conditions (i) and (ii) of (4.5), which completes the proof of the theorem.

Acknowledgements. The authors are grateful to Marcin NowakPrzygodzki for devising a computer program which successfully tested the results of the paper. The program is available at http://www.mif.pg.gda.pl/ homepages/graff/s3.html.

The research was supported by KBN grant No 1 P03A 03929.

\section{References}

[1] I. K. Babenko and S. A. Bogatyi, The behavior of the index of periodic points under iterations of a mapping, Math. USSR-Izv. 38 (1992), 1-26.

[2] R. F. Brown, The Lefschetz Fixed Point Theorem, Scott, Foresman and Co., Glenview, 1971.

[3] S. N. Chow, J. Mallet-Paret and J. A. Yorke, A periodic point index which is a bifurcation invariant, in: Geometric Dynamics (Rio de Janeiro, 1981), Lecture Notes in Math. 1007, Springer, Berlin, 1983, 109-131.

[4] A. Dold, Fixed point indices of iterated maps, Invent. Math. 74 (1983), 419-435.

[5] G. Graff, Minimal number of periodic points for smooth self-maps of two-holed 3dimensional closed ball, Topol. Methods Nonlinear Anal. 33 (2009), 121-130.

[6] G. Graff and J. Jezierski, Minimal number of periodic points for $C^{1}$ self-maps of compact simply-connected manifolds, Forum Math. 21 (2009), 491-509.

[7] G. Graff and P. Nowak-Przygodzki, Fixed point indices of iterations of $C^{1}$ maps in $\mathbb{R}^{3}$, Discrete Contin. Dynam. Systems 16 (2006), 843-856.

[8] M. Greenberg, Lectures on Algebraic Topology, Benjamin, Menlo Park, CA, 1971.

[9] J. Jezierski, Wecken's theorem for periodic points in dimension at least 3, Topology Appl. 153 (2006), 1825-1837. 
[10] J. Jezierski and W. Marzantowicz, Homotopy Methods in Topological Fixed and Periodic Points Theory, Topol. Fixed Point Theory Appl. 3. Springer, Dordrecht, 2005.

[11] B. J. Jiang, Lectures on the Nielsen Fixed Point Theory, Contemp. Math. 14, Amer. Math. Soc., Providence 1983.

[12] J. Llibre, J. Paranõs and J. A. Rodriguez, Periods for transversal maps on compact manifolds with a given homology, Houston J. Math. 24 (1998), 397-407.

[13] W. Marzantowicz and P. Przygodzki, Finding periodic points of a map by use of a $k$-adic expansion, Discrete Contin. Dynam. Systems 5 (1999), 495-514.

[14] M. Shub and P. Sullivan, A remark on the Lefschetz fixed point formula for differentiable maps, Topology 13 (1974), 189-191.

Faculty of Applied

Physics and Mathematics

Gdańsk University of Technology

Narutowicza 11/12

80-233 Gdańsk, Poland

E-mail: graff@mif.pg.gda.pl
Faculty of Applied

Informatics and Mathematics

Warsaw University of Life Sciences (SGGW)

Nowoursynowska 159 00-757 Warszawa, Poland

E-mail: jezierski@acn.waw.pl

Received 19 February 2008;

in revised form 19 May 2009 